\title{
LA TUMBA DEL EMPERADOR JULIANO
}

\author{
JAVIER ARCE MARTINEZ \\ Instituto Rodrigo Caro (C. S. I. C.)
}

\begin{abstract}
El lugar de enterramiento del emperador Juliano ha sido objeto de numerosas controversias ya desde la antigüedad. En este artículo se relacionan las fuentes que tratan del tema, así como las circunstancias en que tuvo lugar la muerte de Juliano y el traslado de su cadáver desde Persia a Tarso y desde aquí a Constantinopla.

The burial place of the emperor Julianus has been the object of a great number of controversies even from antiquity. In this article, the sources dealing with this topic are related, as well as the circumstances surrounding Julianus' death and the transfer of his corpse from Persia to Tarsus and from here to Constantinople.
\end{abstract}

\section{INTRODUCCION}

A la entrada del actual emplazamiento del Museo Arqueológico de Istambul, el visitante puede contemplar una serie de magníficos sarcófagos de pórfido, que con razón han sido identificados como los sepulcros de los emperadores tardo-romanos y bizantinos que originariamente estuvieron en la famosa Iglesia de los Santos Apóstoles, hoy completamente desaparecida. Esta iglesia era de planta cruciforme y su descripción se encuentra tanto en el tratado De Aedificiis de Procopio (I. 4.9-24) del s. VI, cuanto - y esta vez más detalladamente- en Nicolás Mesarites, s. XII, en su Descripción de la Iglesia de los Santos Apóstoles. Poseía dos mausoleos donde se hallaban los sarcófagos imperiales. En una primera fase - como muy bien ha estudiado $\mathrm{Ph}$. Grierson- en uno de ellos, el llamado Mausoleo de Constantino, hubo sólo tres tumbas: la del propio Constantino El Grande (con Helena o Faustina); la de Constancio II, uno de sus hijos, y la de Teodosio I (1). Sabemos que tres Emperadores del s. IV, además de Constantino, Constancio y Teodosio, fueron enterrados en la Iglesia de los Santos Apóstoles, en diferentes momentos: Juliano, Joviano y Valentiniano.

Juliano fue sepultado en Tarso y de allí fue llevado a la capital del Imperio Oriental para ser enterrado. Su traslado tuvo lugar en un momento que discutiremos más adelante; Joviano murió en Dadastana (entre Galatia y Bitinia) y su cuerpo fue también trasladado a Constantinopla; Valentiniano I acabó sus días en la Galia, pero fue también trasladado a la ciudad de Constantino ut... inter divorum reliquias humaretur, como indica Ammiano Marcelino en 30. 10. 1.

(1) Para más detalles sobre la Iglesia y sobre los mausoleos debe consultarse Ph. GRIERSON (1962, 3-63) con la bibliografía fundamental. Igualmente es básico el estudio de G. DOWNEY (1959, 27-51), así como K. WULZINGER (1932, 7-39); R. JANIN (1953, 46-55); J. EBERSOLT (1951, 31-43). 
Valente, Graciano y Valentiniano II, que completan la lista de Emperadores del s. IV, no encontraron, sin embargo, el reposo final junto a sus colegas citados: el cuerpo de Valente no se encontró después de la desastrosa batalla de Adrianópolis, como señala Ammiano. Graciano y Valentiniano II fueron enterrados en Milán.

Cuando muere Joviano en el año 364, en el Mausoleo había pues, solamente, dos sepulcros: el de Constantino y el de su hijo Constancio. Joviano fue, sin duda, en un principio enterrado con ellos ya que, según el texto de Ammiano, su cuerpo se llevó a Constantinopla ut inter Augustorum reliquias conderetur. Poco más tarde y según la sugerente hipótesis de Grierson, Joviano fue desplazado de lugar para dejar sitio a Teodosio. A su vez Joviano fue enterrado entonces en la denominada «stoa» norte de la iglesia y junto a él se colocó el sepulcro del Emperador Juliano, puesto que había sitio suficiente (2). ¿Por qué enterrar juntos a ambos Emperadores? Grierson piensa que, aunque no había entre ellos ningún lazo de consanguinidad, fueron enterrados en el mismo sitio por haber sido anteriormente compañeros de armas y porque Juliano había mantenido en el cargo a Joviano a pesar de ser cristiano. Explicación, a mi entender, un tanto forzada, pero cualquier otro razonamiento lo sería igualmente y es por lo tanto innecesario añadir algo más.

En este trabajo pretendo hacer un análisis detenido de todos los argumentos y datos en pro y en contra para un posible acceso a la identificación segura del sarcófago del Emperador Juliano y al mismo tiempo hago un estudio de las circunstancias y hechos que ocurrieron en la muerte y traslado de cadáver de Juliano de Persia a Tarso y de aquí a Constantinopla. Se analiza además el epigrama que estaba inscrito en su tumba y la problemática de su transmisión literaria.

Debemos en primer lugar recordar brevemente los problemas relativos a la muerte, enterramiento y traslados del cadáver del Emperador.

\section{ENTERRAMIENTO DE JULIANO EN TARSO: CIRCUNSTANCIAS Y DATOS}

El historiador Ammiano Marcelino, que estuvo en la campaña de Persia acompañando a Juliano y que, sin duda, fue también testigo de su muerte, señala que, tras el 26 de junio del 363, última fecha en la vida del joven Emperador, Joviano, su sucesor, envió a Procopio, unido al difunto por lazos de parentesco, a fin de acompañar el cadáver a Tarso donde iba a ser enterrado (3). ¿Por qué a Tarso? Juliano, en efecto, había prometido solemnemente no volver a Antioquía después de la expedición a Persia y había mandado disponerlo todo para establecer su residencia en Tarso: disposuisse enim aiebat, hiemandi gratia per compendiariam viam, consumato procinctu, Tarsum Ciliciae reversurum, scripsisseque ad Memorium praesidem ut in eadem urbe cuncta usui congrua pararentur. Et hoc haud diu postea contigit. Corpus namque eius illuc relatum exequiarum humili pompa, in suburbano sepultum est, ut ipse mandarat (4). Este pasaje

(2) Downey pensó que la «stoa» era un edificio separado de la iglesia principal mientras que Grierson cree que era simplemente una especie de capilla lateral, idea que parece más convincente. Confrontar Ph. GRIERSON (1962, 36 ss.).

(3) Confrontar Ammiano Marcelino (25.9.12). Como Procopio no se unió al grueso del ejército hasta algún tiempo después del 26 de junio el cadáver de Juliano fue embalsamado y transportado con el ejército durante la retirada. Y de hecho asi lo atestigua Efrén Sirio en su Carmina Nisibena, confrontar Ephraem des Syriers Hymnen de Paradiso und contra Julianus, Corpus Scriptorum Orientalum, 79, Lovaina, 1957.

(4) Am. Marc. 23.2.5. Este texto, por otro lado, da una idea bastante clara de la verdadera dimensión que de la campaña tenía en la mente Juliano, ya que, contrariamente a lo que algunos han supuesto, Juliano pretendía regresar a Tarso hiemandi gratia, es decir, a los pocos meses. Su expedición es una expedición de castigo del tipo de las que llevó a cabo en las Galias aunque más aparatosamente montada. 
significa solamente que Juliano había ordenado prepararlo todo en Tarso para su estancia, pero no, como piensa G. Downey, que Juliano hubiese dado órdenes para que se le enterrase allí, ya que el Emperador confiaba y esperaba volver con vida de la expedición (5). En última instancia se podría pensar que lo que Juliano había ordenado es que, si moría, se le enterrase humili pompa, hecho que concuerda bien con la sobriedad y el estilo de vida que gustaba y caracterizaba al Emperador. Ammiano no hace más que constatar aquí una coincidencia tristemente cierta: volvió Juliano a Tarso, como había dispuesto, pero volvió muerto.

Un poco más adelante el mismo historiador señala que Joviano, meses después, de regreso ya de Persia, pasó por Tarso y ordenó adornar el sepulcro de su predecesor: Exindeque egredi nimium properans exornari sepulchrum statuit Iuliani in pomerio situm itineris, quod ad Tauri montis angustias ducit, cuius suprema et cineres, siqui tunc ad perpetuandam gloriam recte factorum praeter lambere Tiberis, intersecans urbem aeternam divorumque veterum monumenta praestingens (Am. Marc. 25.10.5). Varios aspectos importantes ilustra este pasaje que merece, a nuestro propósito, un comentario detallado.

En primer lugar el que se refiere al emplazamiento del sepulchrum de Juliano, que estaba situado - como por otro lado era lo normal- in pomerio itineris (cfr. BESNIER, s. v. Pomerium en DAREMBERG-SAGLIO), lo cual coincide, no sólo con afirmaciones anteriores del propio Ammiano (in suburbano Tarsensi dice en $23.2 .5 \mathrm{y}$

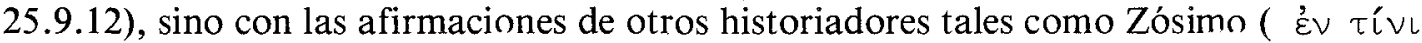

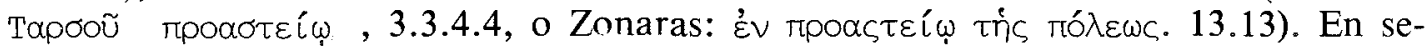
gundo término hay que observar en este pasaje el hecho de que a los pocos meses de ser nombrado Emperador, Joviano mismo sea quien disponga adornar el sepulcro de su predecesor, en este caso un pagano que no merecería tal honor dentro del pensamiento de un emperador cristiano como Joviano, al menos en teoría y a priori. Este texto, efectivamente, no ha sido suficientemente valorado por quienes se han planteado el problema del traslado en la Iglesia de los Santos Apóstoles, bien esto haya tenido lugar en época de Teodosio I bien en tiempos de León I (vid. infra), pues algunos autores consideran un obstáculo - y no el menor - para el traslado a Constantinopla el hecho de que Juliano fuera pagano. En concreto este texto de Ammiano desvirtúa en cierto modo la argumentación de A. A. Vasiliev - basada en una hipotética reconstrucción de los hechos según la cual el epitafio del que más adelante hablaremos no fue puesto sobre el sarcófago del Emperador, sino sobre una piedra o placa adyacente al mismo y ésta, por su indudable carácter laudatorio hacia Juliano, fue considerada inadmisible para Joviano, que la destruyó (VASILIEV, 1948, 1-26, esp. 19). Nada de esto es seguro y, más bien, los indicios llevan a pensar lo contrario. Es cierto, sin embargo, que Joviano pudo preocuparse por el sepulcro de Juliano por imperativos políticos, a fin de congraciarse con los partidarios de éste, pero aún así la hipótesis de Vasiliev es prácticamente inadmisible.

El exornari sepulchrum del texto que comentamos es también significativo en otro sentido: por cuanto que permite hacerse una idea y explica cómo llevó a cabo Procopio la misión que le fue encomendada, es decir, con precipitación y desorden: quid ad exsequendum profectus, confestim corpore sepulto discessit (Am. Marc. 25.9.13).

(5) " La opinión de G. DOWNEY $(1959,46)$ es para mí errónea interpretación del $u$ t ipse mandarat. Recientemente R. TURCAN $(1975,125)$ insiste en la idea de que Juliano quiso expresamente ser enterrado en Tarso basándose en Am. Marc. 25.5.1 y 10.5, y lo pone en relación con los posibles defensores del mitraísmo del Emperador. Pero en 25.5.1 Ammiano vuelve a ser ambiguo refiriéndose como en 23.2.5 al hecho de que había ordenado volver a Tarso, pero vivo, no muerto; 25.10 .5 no dice más que Juliano está enterrado en esta ciudad. 
Da idea también el texto de que el prestigio de Juliano, último representante de la dinastía constantiniana y al fin y al cabo y pese a cristianos o fanáticos, Emperador de Roma, no se eclipsó fácilmente a pesar de los denodados esfuerzos de los cristianos para que así fuera (6).

Aún se pueden sacar otras conclusiones del texto citado de Ammiano. La más obvia e importante de ellas es de orden cronológico y fue $\mathrm{Ph}$. Grierson quien la puso de relieve con anterioridad. Queda claro que cuando Ammiano está escribiendo (más propiamente recitando a su auditorio romano), Juliano seguía aún enterrado en Tarso y no se había llevado a cabo su traslado a Constantinopla. El libro XXV de Ammiano fue escrito probablemente en 390-391 y recitado en Roma en plena época teodosiana (MAENCHEN-HELFEN, 1955, 384; PIGHI, IX -que fecha en 391-392-; CAMERON, 1971, 255 ss.). El traslado del Emperador tuvo que ocurrir forzosamente con posterioridad a esa fecha y quizás - como piensa Grierson- como consecuencia precisamente de esta apelación pública del gran historiador (7). Siendo esto perfectamente posible, R. Calza ha propuesto otra fecha para el traslado que parece bastante convincente ya que piensa que pudo ser hecho, como consecuencia del edicto de León I (13 del 8 del 457$)$ que requería una digna sepultura para el último emperador de la dinastía constantiniana (CALZA, 1973, 366, n. 7; El edicto de León I en CJ 1.5.9). Ambos argumentos, pero sobre todo y sin duda alguna el primero, invalidan la reciente afirmación de C. C. B. Amstrong, según la cual el círculo de amigos de Juliano en Efeso y concretamente el gobernador Clerco, que tenía cierta influencia sobre Valente, forzaron a éste al traslado a Constantinopla del cadáver del Emperador (8). Sea lo que fuere el hecho es que en 390 Juliano estaba aún enterrado en Tarso y Ammiano, en un rasgo de romanismo, proclamaba que era en Roma, en la Roma Aeterna, donde debería estar enterrado y no en Tarso. Por contraste, Libanio, escribiendo en 365, pensaba que era en Atenas, junto a la Academia, al lado de Platón, el lugar donde debía reposar Juliano (Or. 18.306; NORMAN, XXXIV). De las dos opiniones de estos dos grandes panegiristas de Juliano es posible comprender la distinta visión que ambos tenían de su héroe: Ammiano veía en él antes que nada al Emperador romano, militar, que merecía estar asociado para siempre con las hazañas de la Ciudad Eterna y sus tradiciones; Libanio entendía, por su parte, mucho más el aspecto filosófico e intelectual de Juliano y su vinculación al neoplatonismo. Por azar Juliano no fue enterrado en ninguna de las dos ciudades que sus panegiristas propugnaban, sino en Constantinopla, capital del Imperio Oriental, donde probablemente no hubiera deseado estar.

Para finalizar el comentario a este significativo pasaje de Ammiano sólo una observación: la precisión in pomerio situm itineris quod ad Tauri montis angustias ducit, ha-

(6) Confrontar Eutr. 10.16; Libanio, Or. 18.304; Eunapio, fr. 23; A. D. NOCK (1957, 115 ss.) que estudia el pasaje de Libanio citado; J. STRAUB $(1962,310-326)$. Recientemente R. CALZA $(1973,388)$ rechaza la identificación de Juliano en su apoteosis en el famoso díptico del Museo Británico, entre otras razones porque «non risulta che i successori di Giuliano (Gioviano, Valentiniano, Valente) abbiano avuto cura di promovere l'apoteosi». Esto es cierto, al menos en parte, pero la argumentación no es suficiente, porque por ejemplo Archer ST. CLAIRE (1964, 205 ss., esp. 290) demuestra con razones mejores que el representado en el dístico es Juliano. R. Calza no menciona este importante estudio. Por otro lado y además, Gregorio Nazianceno nos habla de un templo dedicado al culto de Juliano erigido en Tarso (LIBANIO, V. 8).

(7) Ph. GRIERSON (1962, 40-41). Este autor cree que el silencio de Zósimo (s. VI) al respecto no es relevante y piensa que precisamente por ser pagano (como su fuente Eunapio de Sardes) no quiso mencionar el traslado a la capital cristiana. La apelación de Ammiano pudo llegar a Teodosio durante su visita a Italia en 391-392.

(8) Confrontar Proceedings of Classical Association, 1972, vol. LXIX, 30, con un resumen del trabajo de C. C. B. AMSTRONG. 
ce pensar en la posibilidad de que el propio Ammiano visitase personalmente la tumba de su admirado Juliano (9).

El relato del traslado del cadáver de Juliano lo hallamos también en Zósimo, expresado de forma más escueta y concisa (Zos. 3.34), quien dice que, en compañía de su guardia personal, el Emperador Joviano llegó a Antioquía, mientras el grueso del ejército acompañó al cadáver de Juliano a Tarso. R. T. Ridley ha querido ver en esta afirmación un error, ya que Ammiano no menciona al ejército como acompañante, sino sólo a Procopio (RIDLEY, 1972, 277 ss., esp. 293 ss.). Nada impide pensar - sin embargoque acompañase a Procopio un gran contingente de tropas, tanto de seguidores fieles a Juliano cuanto de otros deseosos ya de regresar tras la penosa campaña. Zósimo continúa diciendo que el cuerpo de Juliano fue enterrado en un sarcófago en el que se grabó

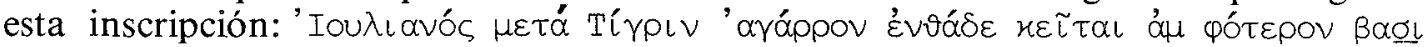

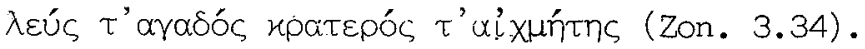

I. Zonaras, siglos más tarde, recoge en su Epitome Historiarum la misma versión de los hechos añadiendo, sin embargo, algún dato fundamental. Repite que fue el ejército el que acompañó el cadáver a Tarso y señala que fue enterrado en un barrio de la ciudad (Zonaras sigue aquí, sin duda, la misma fuente que Zósimo, esto es, Eunapio), y añade que en su sarcófago se inscribió un epigrama de cuatro versos. Añade el historiador que más tarde, sin precisión cronológica alguna, fue trasladado a Constantinopla (Zon. 13.13).

Es el momento ahora de hacer frente, vistos los datos de las fuentes literarias, a dos problemas: 1) el problema de la inscripción o epitafio y 2) el problema del sarcófago donde fue inscrito.

\section{EL EPITAFIO DEL EMPERADOR JULIANO}

Hemos visto que tanto Zonaras como Zósimo dan el texto de la inscripción que se puso en el sarcófago de Juliano. Lo primero que salta a la vista es que ambos presentan diversas versiones de la misma: en Zósimo es un dístico y en Zonaras son cuatro versos, siendo el último idéntico en ambas versiones. El dístico de Zósimo fue recogido en la Antología Palatina (ed. Waltz) 7.747 y fue atribuido a Libanio. Esta atribución fue negada hace tiempo por Förster (10).

En el lemma del epigrama no aparece ninguno de los apelativos que normalmente se asignan a Juliano en la Antología (Anth. Pal. 14.148 y 11.108) y el dístico, formado por dos hexámetros dactílicos, tiene evidentes recuerdos homéricos (Il. III, $179 \mathrm{y} \mathrm{II,}$ 845). La traducción del primer verso, sin embargo, ha sido interpretada de diversas formas. Yo creo que la más apropiada podría ser ésta: «Juliano, que pasó el Tigris de rápida corriente, yace aquí enterrado». La razón es obvia: si el epitafio se inscribió en el sar-

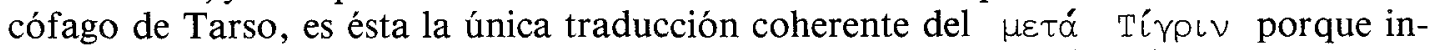
terpretar $\mu \varepsilon \tau \alpha ́$ como «al otro lado del Tigris», como pretenden algunos, sería una

(9) Después de su participación en la expedición a Persia Ammiano va a Antioquía. Confrontar 29.1.24 ss.

(10) Confrontar FÖRSTER (1876, 213-214). La identificación es rechazada también por Waltz y Beckby en sus ediciones del epigrama en la Antología. 
contradicción (11). Hemos visto ya que estos dos versos aparecen por primera vez en Zósimo. El primer punto a resolver es saber de dónde tomo Zósimo esta información.

Es bien sabida la problemática que encierra un autor como Zósimo cuyo sentido de la exactitud histórica deja mucho que desear (12). Esta inexactitud que caracteriza a Zósimo se agudiza, si cabe, en el libro III y concretamente en el relato de la campaña persa; dilucidar cuál es la fuente (o fuentes) que sigue para este libro sigue siendo causa de interminable controversia entre los especialistas (v. PASCHOUD, I, XLIII), aunque se puede afirmar con certeza que normalmente Zósimo sigue a Eunapio y, en el caso concreto que nos ocupa, esto es, en la transmisión del epitafio que luego pasará a la Antología, cabe pensar con bastante probabilidad que Zósimo seguía a Eunapio (13). Aunque poseemos muy fragmentariamente la obra de este historiador, se observa en sus Historias una cierta tendencia a consignar oráculos, versos o textos similares referentes al Emperador (ARCE, 1975, 94 ss.). No tendría nada de extraño que Eunapio, pues, hubiera recogido también el epitafio con anterioridad (14). El tono homérico del mismo -muy en consonancia con los gustos de Juliano- hace pensar que fuera redactado por alguno de sus amigos íntimos, quizás Oribasio mismo (15). El autor debió conocer directamente la tumba y en este caso lo transmitido por Zósimo sería la versión más exacta. Sin embargo no hay más remedio que dudar de su completa autenticidad, ya que parece que Zósimo resumió de alguna forma el original, pues, como hemos visto, Zonaras -que deriva de una fuente indudablemente pagana y probablemente de Eunapio (16)da una versión más extensa del epitafio, versión que el cronógrafo bizantino Cedrenos recoge igualmente (I, 539). Sin duda el transmitido por estos dos últimos autores debió de ser el original y lo conservado en Zósimo puede ser un resumen del texto inicial en el que procuró mantener la idea central de la campaña persa y el último verso que condensaba muy bien las virtudes del Emperador de acuerdo con la propia visión de Zósimo sobre él.

\section{EL SARCOFAGO DEL EMPERADOR JULIANO}

El problema se centra ahora en el sarcófago donde fue grabada la inscripción. Sería lógico pensar que si se hubiese hallado dicho sarcófago apareciera en él la inscripción que, tanto Zósimo como Zonaras y Cedrenos, dicen que se inscribió aunque den versiones distintas de su contenido.

(11) Beckby traduce: «Hinter dem reissenden Tigris liegt hier Julianus begraden»; Waltz: «Julien, aprés avoir franchi le Tigre au cours rapide, est ici couché»; Platón (Loeb): «Julian lies here on the further bank of the strong current of Tigris»; PEECK (1960, 214): «Julian, der den breitströmenden Tigris überschift, liegt hier nun begraden»; BIDEZ (1930, 330): «des bords du Tigris impétueux Julien est venu dormir

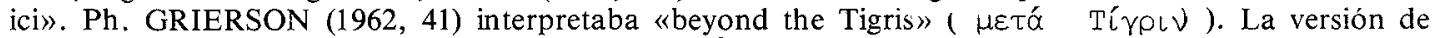

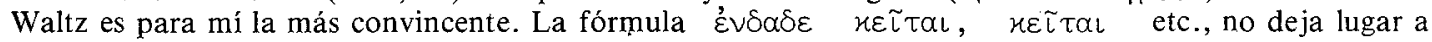
dudas. Confrontar $M A M A$, VII, n. ${ }^{\circ} 104$ a-b-c-d; VIII, $144 ; 155 ; 317 ; 272$.

(12) A. CAMERON (1969, 106-110) para la fecha; F. PASCHOUD (I, XVII); sobre los numerosos errores de Zósimo - además de la introducción de F. Paschoud a su edición y sus notas- confrontar R. T. RIDLEY (1972, 277 ss.) y DILLEMANN (1961, 87 ss., esp. 144 ss.). Puede verse también J. ARCE (1975, 138 ss.).

(13) Eunapio a su vez seguía a Oribasio. Confrontar F. PASCHOUD (I, LV).

(14) Para esta tendencia de Eunapio confrontar fr. 27.24.26 (FHG, Müller, vol. IV).

(15) Para los gustos y aficiones homéricos en Juliano confrontar Jul. 112 C; 351 C (BIDEZ, 1930); Am. Marc. 16.7.6; Lib. Or. 12.27; $\operatorname{BIDEZ~(1930,~21),~etc.~}$

(16) BIDEZ (1930, 338); P. ALLARD (1910, III, 399); K. ZIEGLER (XA, 729) declara simplemente que por el libro XIII la fuente es desconocida. 


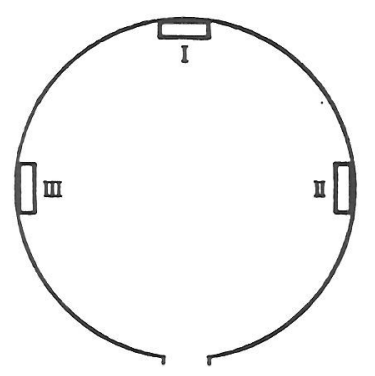

Stage $A$

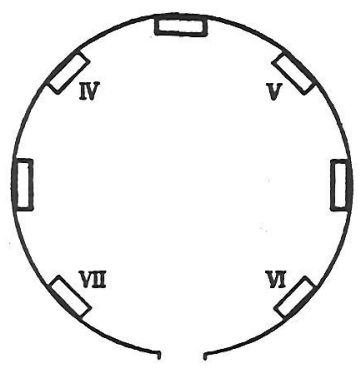

Stage $B$

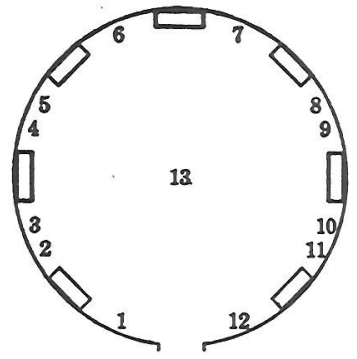

Stage $C$

Figura 1.-Planta esquemática del mausoleo de Constantino.

En la descripción que hace el Libro de las Ceremonias (II, 42) del sarcófago de Juliano en la Iglesia de los Santos Apóstoles, se habla de su forma ru

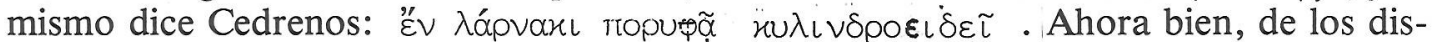
tintos sarcófagos de pórfido que están hoy en la entrada del Museo Arqueológico de Istambul y en el patio de Sta. Irene que pertenecieron al Mausoleo de la Iglesia de los Santos Apóstoles, sólo hay uno que responde de alguna manera a esas características -esto es, que sea de forma cilíndrica o redondeada - por tener ambos extremos semicirculares (f. 1). Este sarcófago fue identificado por Ebersolt y luego por R. Delbrueck como el sarcófago del Emperador Juliano (17). La dificultad para esta identificación (el propio Ebersolt - 1921, 13 - ya dudaba debido a la inexactitud de los autores bizantinos) reside, a mi entender, en que en él no hay inscripción ni rastro de ella, además de que el calificativo de "de forma cilíndrica» puede ser discutible respecto a la propiedad del término. Para salvar el inconveniente de la no existencia de inscripción Vasiliev estableció la siguiente ingeniosa hipótesis: la inscripción se grabó no sobre el sarcófago, sino sobre una piedra o placa adyacente al mismo (fenómeno, por otro lado, ni imposible ni infrecuente y que se usaba para diferenciar un sarcófago; sobre ello véase GRIERSON, $1962,9)$. Más tarde - continúa Vasiliev- Joviano, al comprobar el tono laudatorio de la inscripción, la consideró inadmisible y la mandó quitar. De aquí que por ello no haya que esperar ninguna inscripción en el sarcófago de Constantinopla (VASILIEV, 1948, 20).

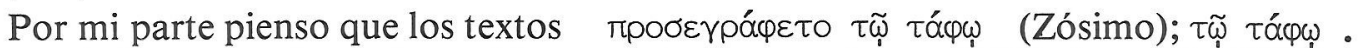

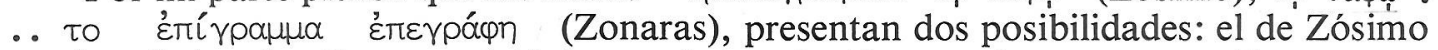
puede referirse (por la preposición) a que la inscripción era adyacente, pero Zonaras parece indicar que se hizo encima (la posibilidad de que se haya pintado la inscripción no se excluye del significado del verbo). Sin embargo el exornari no deja lugar a dudas respecto a la acción de Joviano que hizo justamente lo contrario que propone Vasiliev.

A. Maricq propuso otra solución al problema partiendo de la base de que la identificación hecha por Delbrueck era correcta: el cronógrafo Cedrenos - que es quien señala que en el sarcófago de Constantinopla se grabó la inscripción-cometió una equivo-

(17) J. EBERSOLT (1921, 6, 11, 13, 1.XII); R. DELBRUECK (1932, 14 y 214). R. Delbrueck cree que se trata no obstante de un sarcófago tolemaico trasladado de Alejandría a Constantinopla para sepultar a Juliano: «Es ist, wie gesagt, vielleicht ein ptolemaisches Stück und nicht für Julianus gearbeitet», esto es, el sarcófago no fue hecho expresamente para Juliano.

(18) Cedrenos, en efecto, es el único que dice que «fue trasladado (su cuerpo) a Constantinopla y colocado en un sarcófago de pórfido cilíndrico sobre el cual se inscribió el siguiente epigrama..., etc.». El texto es ambiguo porque Cedrenos está tomando su información de otros autores -en este caso de Leo Grammatico- quienes a su vez confunden y mezclan sus datos. Confrontar A. MARICQ (1952, 320-321). 


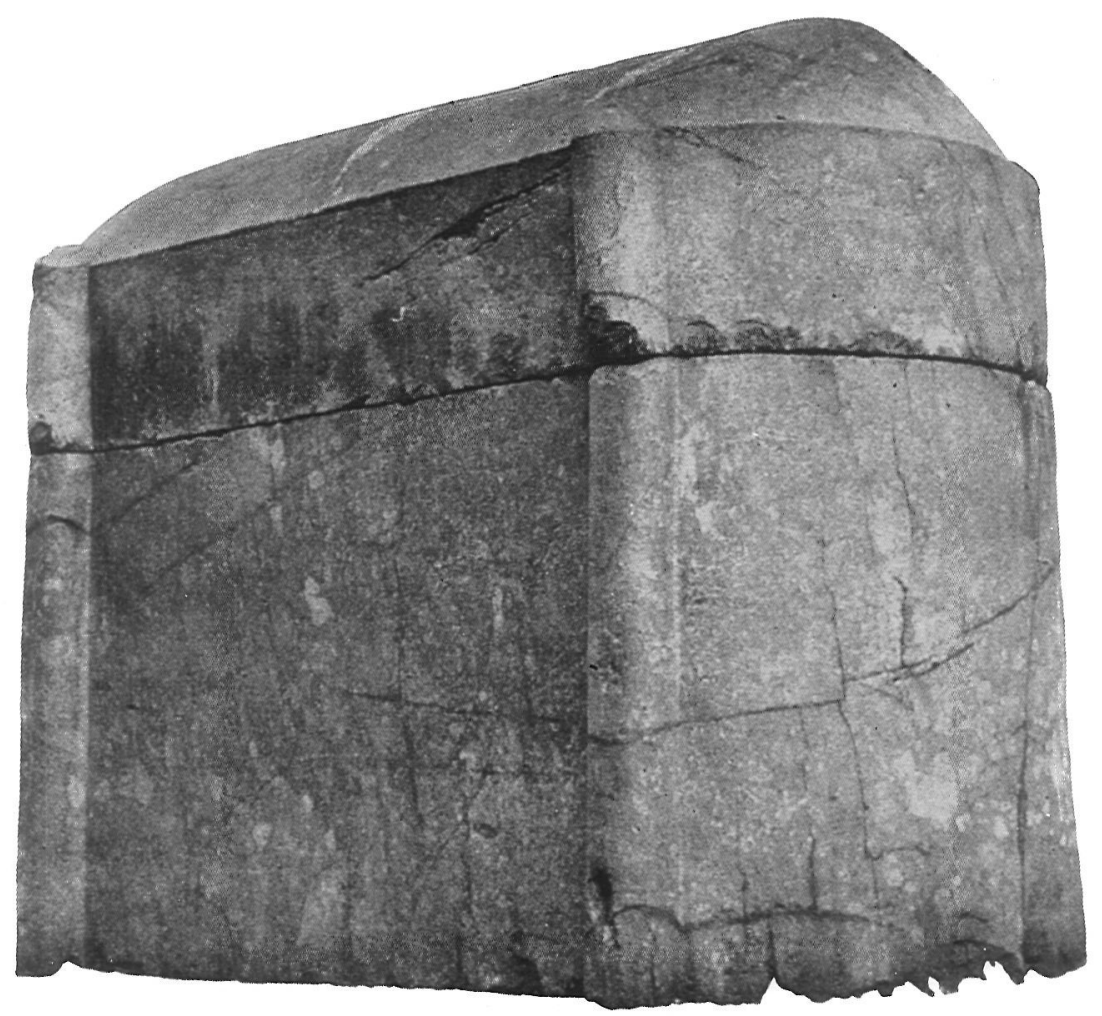

Figura 2.-Sarcófago de Constantinopla.

cación debido a su peculiar forma de redactar y lo que dice como grabado en el sarcófago de Constantinopla es falso y que sólo se refería a lo que se grabó en el de Tarso, que resultaba ser distinto que el de la Iglesia de los Santos Apóstoles donde fue depositado luego; por lo tanto - concluye Maricq-, «no hay motivos para extrañarse de la ausencia de restos de inscripción en el sarcófago de Constantinopla» (18). Ph. Grierson cree por su parte que el sarcófago cilíndrico que normalmente se atribuye a Juliano puede ser el suyo, aunque admite la posibilidad de que sea otro, publicado y estudiado por C. Mango. Rechaza la hipótesis de Maricq señalando que para él los textos de Zonaras y Cedrenos son suficientemente claros (lo cual es válido en todo para el de Cedrenos y de ninguna manera para el de Zonaras), aunque reconoce que no se puede dar crédito total a los cronistas bizantinos cuando mencionan inscripciones. Se inclina a creer que el epitafio de Zonaras/Cedrenos es el verdadero considerando que el transmitido por Zósimo es una cita de memoria (19). Por último, Cyril Mango, estudiando la descripción que dio de los sarcófagos descubiertos en Constantinopla en 1750 por el mercader francés J. C. Flachat (f. 2), llega a la conclusión de que puesto que uno de ellos es cilíndrico y de pórfido, éste podría ser, quizá con más probabilidad, el de Juliano a pesar de que lleve inscrita una cruz en uno de los lados. Este sarcófago podría haber llevado la inscripción, aunque resulta muy extraño que Flachat no la hubiese registrado en su descripción (MANGO, 1962, 401).

(19) Ph. GRIERSON $(1962,41)$. La poca credibilidad de los autores bizantinos citando inscripciones analizada en G. DOWNEY (1935, 55-72). Ph. Grierson piensa a propósito del epitafio citado en Zósimo en una segunda posibilidad: esto es, que fuera «a first draft of the epigramm formulated when the news of Julian's death had reached his friends and nothing had yet been determined regarding his place of burial». Esta hipótesis está hecha en base a una interpretación del epigrama que yo creo equivocada. 


\section{CONCLUSION}

Expuesta ya la problemática es conveniente ahora hacer un resumen de lo dicho para llegar a establecer unas posibles conclusiones.

Hemos dejado establecido que Juliano fue transportado desde el lugar de su muerte a Tarso porque él había expresado su deseo de residir en esta ciudad a la vuelta de la campaña y no porque hubiera ordenado expresamente que se le enterrase allí. Acompañó su cadáver Procopio, al parecer con parte de sus tropas. Su enterramiento fue precipitado debido a la prisa de Procopio en desaparecer u ocultarse. En su sarcófago se grabó un epitafio que fue recogido por Oribasio (si es que no lo redacto él mismo). Eunapio lo tomó de Oribasio y Zósimo lo repite resumiendo la idea central del mismo. El compilador del libro VII de la Antología (o uno de ellos) - como señaló ya Preger-habiendo leído a Zósimo, incluyó los versos atribuyéndoselos «sua sponte» a Libanio. Zonaras recoge el epitafio in extenso que se conservó igualmente en Cedrenos. Según Zósi-

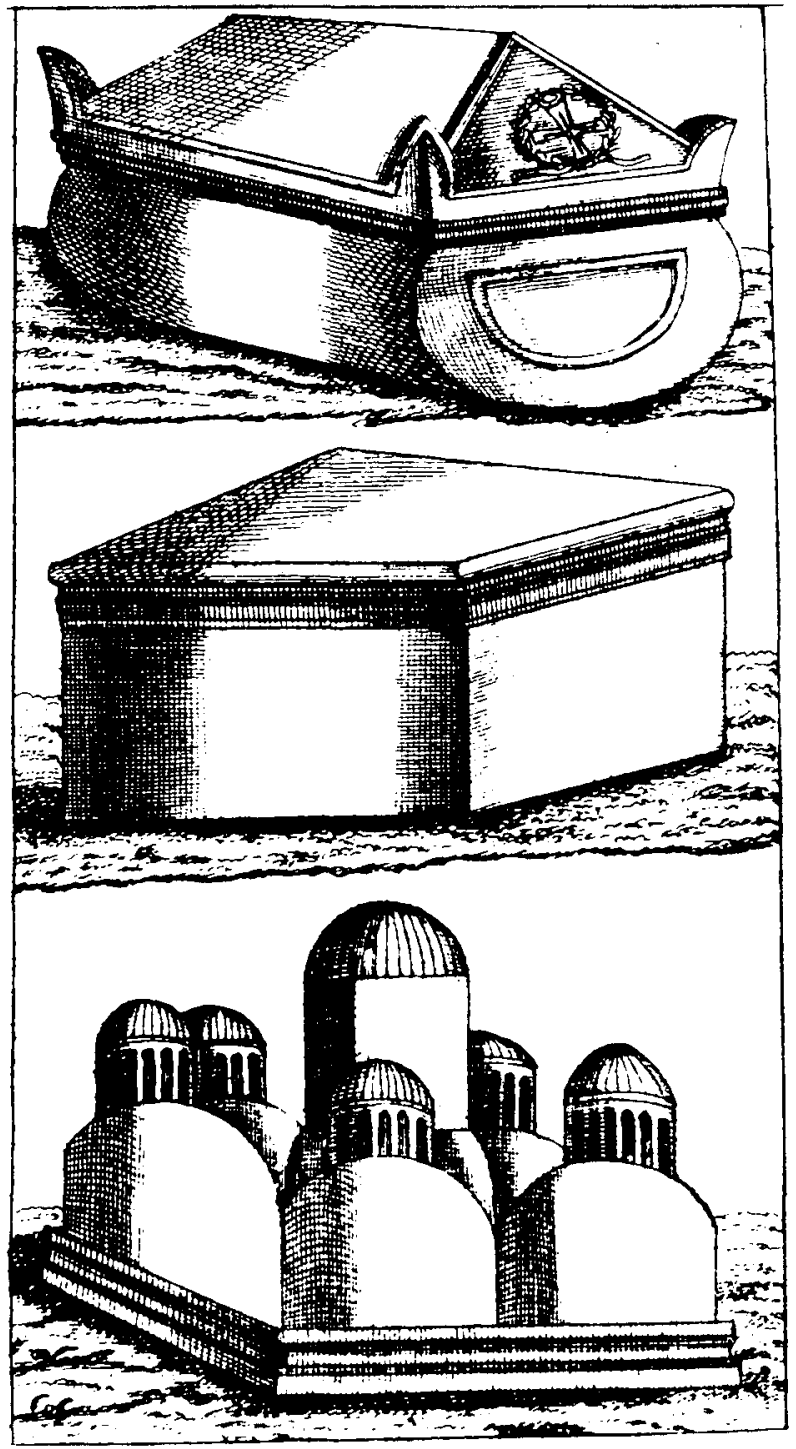

Figura 3.-Sarcófagos bizantinos. 
mo y Zonaras el epitafio se grabó en el sepulcro o sarcófago de Tarso. Según Cedrenos estaba, al menos, en el de Constantinopla. Ahora bien, si el sarcófago de Constantinopla que pasa por ser el de Juliano es el mismo que el de Tarso, tendría que conservar entonces la inscripción. Pero ello no es así, y por lo tanto hay que considerar las siguientes posibilidades:

a) Que la inscripción no fuera escrita en el sarcófago sino en una piedra o placa adyacente al mismo (Vasiliev).

b) Que el sarcófago de Tarso no fue el mismo que el de Istambul, y por eso no aparece en él la inscripción (Maricq). Esto implicaría que Cedrenos no es exacto en su relato, lo cual es altamente posible y lícito de suponer.

c) Que el sarcófago de Istambul no tenga en absoluto nada que ver con Juliano (en realidad la expresión $\quad x u \lambda \iota v \delta p o \varepsilon \iota \delta n ́ s$ no se aviene perfectamente con el sarcófago supuesto), y que, en cambio, sea el descrito por Flachat, descubierto en 1750 , de forma cilíndrica también. Puesto que éste no se conserva (MANGO, $1962,400)$ no podemos saber si llevaba o no la inscripción. Flachat al menos no dice nada de ella en su descripción, lo cual es significativo, pues si hubiera existido la hubiera descrito también.

Por lo tanto y puesto que la hipótesis de Vasiliev es insostenible -como hemos dicho- hay que considerar que Maricq está en lo cierto al afirmar que hubo al menos dos sarcófagos para Juliano: uno, el de Tarso, desaparecido y con la inscripción-epitafio; el otro, el de pórfido de forma cilíndrica sin inscripción. Abonaría esta hipótesis el hecho de la aseveración de Delbrueck de que el de Istambul es un sarcófago tolemaico. Ahora bien, ¿se puede pensar que se trajo un sarcófago de Alejandría a Tarso a los tres meses de su muerte, o es más lógico que esto se hiciera a Constantinopla al cabo de varios años y como consecuencia del traslado solemne del cadáver del Emperador a la capital de la Pars Orientis? Este traslado debió de suceder o en 391-392 -porque en 390 todavía estaba Juliano enterrado en Tarso como se desprende del texto de Ammiano 25.10.5 - o como consecuencia del Edicto de León I recogido en $C J$ I.9.5 en 457.

No obstante, la posibilidad de que el sarcófago descrito por Flachat sea el de Juliano es muy digna de tener en cuenta y tiene a su favor el hecho de que se aviene mejor con la descripción de los historiadores bizantinos. Aunque ello fuera así hay que afirmar que hubo dos sarcófagos para Juliano.

Así pues, el sarcófago que hay a la entrada del Museo Arqueológico de Istambul que reproducimos en la figura 1, puede ser el que tuvo el cuerpo del gran Juliano, pero no es absolutamente seguro. Quizá se haya perdido para siempre como se perdieron todas las ilusiones y energías de aquel hombre extraordinario.

\title{
ABREVIATURAS
}

\author{
$A J P h \quad$ American Journal of Philology. \\ $B Z \quad$ Bizantinische Zeitschrift. \\ CJ Classical Journal. \\ DOP Dumbarton Oaks Papers. \\ FHG C. Müller's Fragmenta Historicorum Graecorum. (París). \\ JHS Journal of Hellenic Studies. \\ JRS Journal of Roman Studies. \\ MAMA W. M. Calder (ed), Monumenta Asiae Minoris Antiqua, Manchester, 1928-1962, vols. VII y VIII. \\ $P W \quad$ Pauly-Wissowa-Kroll's, Real Encyclopädie der Classischen Altertumswissenschaft. \\ TAPA Transactions of the American Philological Association.
}




\section{BIBLIOGRAFIA}

ALLARD, P. 1910: Julien l'Apostat. III, París.

ARCE, J. 1975: Estudios sobre las fuentes literarias, epigráficas y numismáticas para la historia del Emperador Juliano, Tesis Doctoral mecanografiada.

BIDEZ, J. 1930: La vie de l'Empereur Julien, París.

CALZA, R. 1973: Iconografia Romana Imperiale, Roma.

CAMERON, A. 1969: The Date of Zosimus New History, Philologus, 113, 106-110.

- 1971: Ammianus and the Historia Augusta, JRS, 61, 255 ss.

DELBRUECK, R. 1932: Antike Porphywerke, Berlín, T. 107.

DILLEMANN, L. 1961: Ammien Marcellin et les pays de l'Euphrate et du Tigre, Syria, 38, 87-158.

DOWNEY, G. 1935: References to inscriptions in the Chronicle of Malalas, TAPA, 66, 55-72.

- 1959: The Tombs of the Bizantine Emperors at the Church of the Holy Apostles in Constantinople, JHS, 79, 27-51.

EBERSOLT, J. 1951: Constantinople: Recueil d'études d'Archéologie et d'Histoire, París.

EPHRAEM DES SYRIERS, 1957: Hymnen de Paradiso und contra Julianus, Corpus Scriptorum Orientalum, 79, Lovaina.

FÖRSTER, R. 1876: Die Schriftstellen des Libanios, Jahrbücher für Classische Philologie, 22, 213-214.

GRIERSON, Ph. 1962: The Tombs and Obits of the Byzantine Emperors (337-1042), DOP, 16, 3-63.

JANIN, R. 1953: La Géographie ecclésiastique de l'Empire Byzantin, I, París.

MAENCHEN-HELFEN, O. 1955: The Date of Ammianus Marcellinus' Last Books, $A J P h, 76,385$ ss.

MANGO, C. 1962: Three Imperial Byzantine Sarcophagi discovered in 1750, DOP, 16, 401.

MARICQ, A. 1952: Notes Philologiques: 4. Les sarcophages impériaux de Constantinople, Bizantion, 22, 320-321.

NOCK, A. D. 1957: Deification and Julian, JRS, 47, 115 ss.

NORMAN, A. F. 1969: Libanius Selected Works, I, Cambridge Mass. (Loeb Series).

PASCHOUD, F. 1971: Zosime. Histoire Nouvelle. I, París.

PEECK, W. 1960: Griechische Grabgedichte, Berlín.

PIGHI, G. B. 1935: Ammiani Marcellini Rerum Gestarum, Milán.

PROCEEDINGS of the Classical Association, vol. LXIX, 1972.

RIDLEY, R. T. 1972: Zosimus the Historian, BZ, 65, 277 ss.

ST. CLAIRE, A.: The Apotheosis Diptych, Art Bulletin, 205 ss.

STRAUB, J. 1962: Die Himmelfahrt des Julianus Apostata, Gymnasium, LXIX, 310-326.

TURCAN, R. 1975: Mithras Platonicus. Recherches sur l'Héllénisation philosophique de Mithra, Leiden. VASILIEV, A. A. 1948: Imperial Porphyry Sarcophagi in Constantinople, DOP, 1-26.

WULZINGER, K. 1932: Die Apostelkirche und die Mehmedije zu Konstantinopel, Byzantion, 7, 7-39. 